\title{
Robust Machine Translation Evaluation with Entailment Features*
}

\author{
Sebastian Padó \\ Stuttgart University \\ pado@ims.uni-stuttgart.de
}

\author{
Michel Galley, Dan Jurafsky, Chris Manning \\ Stanford University \\ \{mgalley,jurafsky,manning\}@ stanford.edu
}

\begin{abstract}
Existing evaluation metrics for machine translation lack crucial robustness: their correlations with human quality judgments vary considerably across languages and genres. We believe that the main reason is their inability to properly capture meaning: A good translation candidate means the same thing as the reference translation, regardless of formulation. We propose a metric that evaluates MT output based on a rich set of features motivated by textual entailment, such as lexical-semantic (in-)compatibility and argument structure overlap. We compare this metric against a combination metric of four state-of-theart scores (BLEU, NIST, TER, and METEOR) in two different settings. The combination metric outperforms the individual scores, but is bested by the entailment-based metric. Combining the entailment and traditional features yields further improvements.
\end{abstract}

\section{Introduction}

Constant evaluation is vital to the progress of machine translation (MT). Since human evaluation is costly and difficult to do reliably, a major focus of research has been on automatic measures of MT quality, pioneered by BLEU (Papineni et al., 2002) and NIST (Doddington, 2002). BLEU and NIST measure MT quality by using the strong correlation between human judgments and the degree of $n$-gram overlap between a system hypothesis translation and one or more reference translations. The resulting scores are cheap and objective.

However, studies such as Callison-Burch et al. (2006) have identified a number of problems with BLEU and related $n$-gram-based scores: (1) BLEUlike metrics are unreliable at the level of individual sentences due to data sparsity; (2) BLEU metrics can be "gamed" by permuting word order; (3) for some corpora and languages, the correlation to human ratings is very low even at the system level; (4) scores are biased towards statistical MT; (5) the quality gap between MT and human translations is not reflected in equally large BLEU differences.

${ }^{*}$ This paper is based on work funded by the Defense Advanced Research Projects Agency through IBM. The content does not necessarily reflect the views of the U.S. Government, and no official endorsement should be inferred.
This is problematic, but not surprising: The metrics treat any divergence from the reference as a negative, while (computational) linguistics has long dealt with linguistic variation that preserves the meaning, usually called paraphrase, such as:

(1) HYP: However, this was declared terrorism by observers and witnesses.

REF: Nevertheless, commentators as well as eyewitnesses are terming it terrorism.

A number of metrics have been designed to account for paraphrase, either by making the matching more intelligent (TER, Snover et al. (2006)), or by using linguistic evidence, mostly lexical similarity (METEOR, Banerjee and Lavie (2005); MaxSim, Chan and $\mathrm{Ng}(2008)$ ), or syntactic overlap (Owczarzak et al. (2008); Liu and Gildea (2005)). Unfortunately, each metrics tend to concentrate on one particular type of linguistic information, none of which always correlates well with human judgments.

Our paper proposes two strategies. We first explore the combination of traditional scores into a more robust ensemble metric with linear regression. Our second, more fundamental, strategy replaces the use of loose surrogates of translation quality with a model that attempts to comprehensively assess meaning equivalence between references and MT hypotheses. We operationalize meaning equivalence by bidirectional textual entailment (RTE, Dagan et al. (2005)), and thus predict the quality of MT hypotheses with a rich RTE feature set. The entailment-based model goes beyond existing word-level "semantic" metrics such as METEOR by integrating phrasal and compositional aspects of meaning equivalence, such as multiword paraphrases, (in-)correct argument and modification relations, and (dis-)allowed phrase reorderings. We demonstrate that the resulting metric beats both individual and combined traditional MT metrics. The complementary features of both metric types can be combined into a joint, superior metric. 

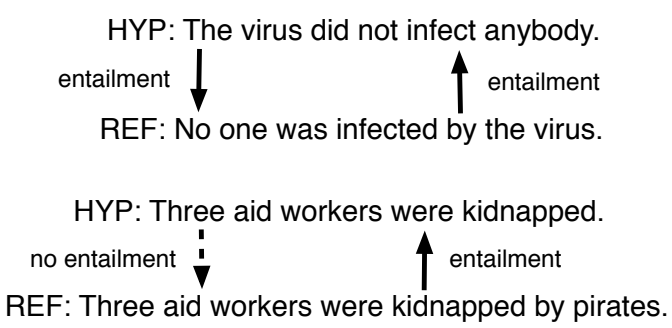

Figure 1: Entailment status between an MT system hypothesis and a reference translation for equivalent (top) and non-equivalent (bottom) translations.

\section{Regression-based MT Quality Prediction}

Current MT metrics tend to focus on a single dimension of linguistic information. Since the importance of these dimensions tends not to be stable across language pairs, genres, and systems, performance of these metrics varies substantially. A simple strategy to overcome this problem could be to combine the judgments of different metrics. For example, Paul et al. (2007) train binary classifiers on a feature set formed by a number of MT metrics. We follow a similar idea, but use a regularized linear regression to directly predict human ratings.

Feature combination via regression is a supervised approach that requires labeled data. As we show in Section 5, this data is available, and the resulting model generalizes well from relatively small amounts of training data.

\section{Textual Entailment vs. MT Evaluation}

Our novel approach to MT evaluation exploits the similarity between MT evaluation and textual entailment (TE). TE was introduced by Dagan et al. (2005) as a concept that corresponds more closely to "common sense" reasoning patterns than classical, strict logical entailment. Textual entailment is defined informally as a relation between two natural language sentences (a premise $\mathrm{P}$ and a hypothesis $\mathrm{H}$ ) that holds if "a human reading $\mathrm{P}$ would infer that $\mathrm{H}$ is most likely true". Knowledge about entailment is beneficial for NLP tasks such as Question Answering (Harabagiu and Hickl, 2006).

The relation between textual entailment and MT evaluation is shown in Figure 1. Perfect MT output and the reference translation entail each other (top). Translation problems that impact semantic equivalence, e.g., deletion or addition of material, can break entailment in one or both directions (bottom).

On the modelling level, there is common ground between RTE and MT evaluation: Both have to distinguish between valid and invalid variation to determine whether two texts convey the same information or not. For example, to recognize the bidirectional entailment in Ex. (1), RTE must account for the following reformulations: synonymy (However/Nevertheless), more general semantic relatedness (observers/commentators), phrasal replacements (and/as well as), and an active/passive alternation that implies structural change (is declared/are terming). This leads us to our main hypothesis: RTE features are designed to distinguish meaning-preserving variation from true divergence and are thus also good predictors in MT evaluation. However, while the original RTE task is asymmetric, MT evaluation needs to determine meaning equivalence, which is a symmetric relation. We do this by checking for entailment in both directions (see Figure 1). Operationally, this ensures we detect translations which either delete or insert material.

Clearly, there are also differences between the two tasks. An important one is that RTE assumes the well-formedness of the two sentences. This is not generally true in MT, and could lead to degraded linguistic analyses. However, entailment relations are more sensitive to the contribution of individual words (MacCartney and Manning, 2008). In Example 2, the modal modifiers break the entailment between two otherwise identical sentences:

(2) HYP: Peter is certainly from Lincolnshire. REF: Peter is possibly from Lincolnshire.

This means that the prediction of TE hinges on correct semantic analysis and is sensitive to misanalyses. In contrast, human MT judgments behave robustly. Translations that involve individual errors, like (2), are judged lower than perfect ones, but usually not crucially so, since most aspects are still rendered correctly. We thus expect even noisy RTE features to be predictive for translation quality. This allows us to use an off-the-shelf RTE system to obtain features, and to combine them using a regression model as described in Section 2.

\subsection{The Stanford Entailment Recognizer}

The Stanford Entailment Recognizer (MacCartney et al., 2006) is a stochastic model that computes match and mismatch features for each premisehypothesis pair. The three stages of the system are shown in Figure 2. The system first uses a robust broad-coverage PCFG parser and a deterministic constituent-dependency converter to construct linguistic representations of the premise and 


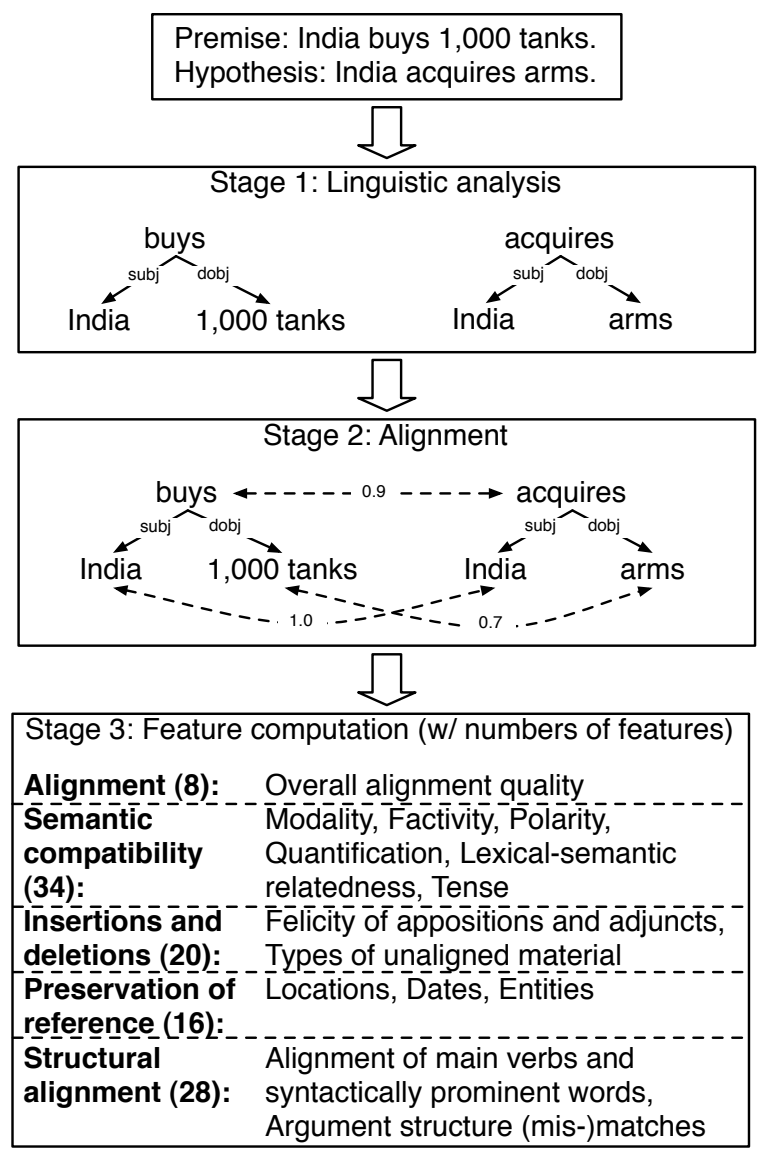

Figure 2: The Stanford Entailment Recognizer

the hypothesis. The results are typed dependency graphs that contain a node for each word and labeled edges representing the grammatical relations between words. Named entities are identified, and contiguous collocations grouped. Next, it identifies the highest-scoring alignment from each node in the hypothesis graph to a single node in the premise graph, or to null. It uses a locally decomposable scoring function: The score of an alignment is the sum of the local word and edge alignment scores. The computation of these scores make extensive use of about ten lexical similarity resources, including WordNet, InfoMap, and Dekang Lin's thesaurus. Since the search space is exponential in the hypothesis length, the system uses stochastic (rather than exhaustive) search based on Gibbs sampling (see de Marneffe et al. (2007)).

Entailment features. In the third stage, the system produces roughly 100 features for each aligned premise-hypothesis pair. A small number of them are real-valued (mostly quality scores), but most are binary implementations of small linguistic theories whose activation indicates syntactic and se- mantic (mis-)matches of different types. Figure 2 groups the features into five classes. Alignment features measure the overall quality of the alignment as given by the lexical resources. Semantic compatibility features check to what extent the aligned material has the same meaning and preserves semantic dimensions such as modality and factivity, taking a limited amount of context into account. Insertion/deletion features explicitly address material that remains unaligned and assess its felicity. Reference features ascertain that the two sentences actually refer to the same events and participants. Finally, structural features add structural considerations by ensuring that argument structure is preserved in the translation. See MacCartney et al. (2006) for details on the features, and Sections 5 and 6 for examples of feature firings.

Efficiency considerations. The use of deep linguistic analysis makes our entailment-based metric considerably more heavyweight than traditional MT metrics. The average total runtime per sentence pair is 5 seconds on an AMD 2.6GHz Opteron core - efficient enough to perform regular evaluations on development and test sets. We are currently investigating caching and optimizations that will enable the use of our metric for MT parameter tuning in a Minimum Error Rate Training setup (Och, 2003).

\section{Experimental Evaluation}

\subsection{Experiments}

Traditionally, human ratings for MT quality have been collected in the form of absolute scores on a five- or seven-point Likert scale, but low reliability numbers for this type of annotation have raised concerns (Callison-Burch et al., 2008). An alternative that has been adopted by the yearly WMT evaluation shared tasks since 2008 is the collection of pairwise preference judgments between pairs of MT hypotheses which can be elicited (somewhat) more reliably. We demonstrate that our approach works well for both types of annotation and different corpora. Experiment 1 models absolute scores on Asian newswire, and Experiment 2 pairwise preferences on European speech and news data.

\subsection{Evaluation}

We evaluate the output of our models both on the sentence and on the system level. At the sentence level, we can correlate predictions in Experiment 1 directly with human judgments with Spearman's $\rho$, 
a non-parametric rank correlation coefficient appropriate for non-normally distributed data. In Experiment 2 , the predictions cannot be pooled between sentences. Instead of correlation, we compute "consistency" (i.e., accuracy) with human preferences.

System-level predictions are computed in both experiments from sentence-level predictions, as the ratio of sentences for which each system provided the best translation (Callison-Burch et al., 2008). We extend this procedure slightly because realvalued predictions cannot predict ties, while human raters decide for a significant portion of sentences (as much as $80 \%$ in absolute score annotation) to "tie" two systems for first place. To simulate this behavior, we compute "tie-aware" predictions as the percentage of sentences where the system's hypothesis was assigned a score better or at most $\varepsilon$ worse than the best system. $\varepsilon$ is set to match the frequency of ties in the training data.

Finally, the predictions are again correlated with human judgments using Spearman's $\rho$. "Tie awareness" makes a considerable practical difference, improving correlation figures by $5-10$ points. ${ }^{1}$

\subsection{Baseline Metrics}

We consider four baselines. They are small regression models as described in Section 2 over component scores of four widely used MT metrics. To alleviate possible nonlinearity, we add all features in linear and log space. Each baselines carries the name of the underlying metric plus the suffix $-R .^{2}$

BLEUR includes the following 18 sentence-level scores: BLEU- $n$ and $n$-gram precision scores $(1 \leq n \leq 4)$; BLEU brevity penalty (BP); BLEU score divided by BP. To counteract BLEU's brittleness at the sentence level, we also smooth BLEU- $n$ and $n$-gram precision as in Lin and Och (2004).

NISTR consists of 16 features. NIST- $n$ scores $(1 \leq n \leq 10)$ and information-weighted $n$-gram precision scores $(1 \leq n \leq 4)$; NIST brevity penalty (BP); and NIST score divided by BP.

\footnotetext{
${ }^{1}$ Due to space constraints, we only show results for "tieaware" predictions. See Padó et al. (2009) for a discussion.

${ }^{2}$ The regression models can simulate the behaviour of each component by setting the weights appropriately, but are strictly more powerful. A possible danger is that the parameters overfit on the training set. We therefore verified that the three non-trivial "baseline" regression models indeed confer a benefit over the default component combination scores: BLEU-1 (which outperformed BLEU-4 in the MetricsMATR 2008 evaluation), NIST-4, and TER (with all costs set to 1). We found higher robustness and improved correlations for the regression models. An exception is BLEU-1 and NIST-4 on Expt. 1 (Ar, $\mathrm{Ch}$ ), which perform $0.5-1$ point better at the sentence level.
}

TERR includes 50 features. We start with the standard TER score and the number of each of the four edit operations. Since the default uniform cost does not always correlate well with human judgment, we duplicate these features for 9 non-uniform edit costs. We find it effective to set insertion cost close to 0 , as a way of enabling surface variation, and indeed the new TERp metric uses a similarly low default insertion cost (Snover et al., 2009).

\section{METEORR consists of METEOR v0.7.}

\subsection{Combination Metrics}

The following three regression models implement the methods discussed in Sections 2 and 3.

MTR combines the 85 features of the four baseline models. It uses no entailment features.

RTER uses the 70 entailment features described in Section 3.1, but no MTR features.

MT+RTER uses all MTR and RTER features, combining matching and entailment evidence. ${ }^{3}$

\section{Expt. 1: Predicting Absolute Scores}

Data. Our first experiment evaluates the models we have proposed on a corpus with traditional annotation on a seven-point scale, namely the NIST OpenMT 2008 corpus. ${ }^{4}$ The corpus contains translations of newswire text into English from three source languages (Arabic (Ar), Chinese (Ch), Urdu (Ur)). Each language consists of 1500-2800 sentence pairs produced by 7-15 MT systems.

We use a "round robin" scheme. We optimize the weights of our regression models on two languages and then predict the human scores on the third language. This gauges performance of our models when training and test data come from the same genre, but from different languages, which we believe to be a setup of practical interest. For each test set, we set the system-level tie parameter $\varepsilon$ so that the relative frequency of ties was equal to the training set (65-80\%). Hypotheses generally had to receive scores within $0.3-0.5$ points to tie.

Results. Table 1 shows the results. We first concentrate on the upper half (sentence-level results). The predictions of all models correlate highly significantly with human judgments, but we still see robustness issues for the individual MT metrics.

\footnotetext{
${ }^{3}$ Software for RTER and MT+RTER is available from http://nlp.stanford.edu/software/mteval.shtml.

${ }^{4}$ Available from http://www. nist.gov.
} 


\begin{tabular}{l|ll||llll|ll|l}
\multirow{2}{*}{ Evaluation } & \multicolumn{2}{|c|}{ Data } & \multicolumn{7}{c}{ Metrics } \\
& train & test & BLEUR & METEORR & NISTR & TERR & MTR & RTER & MT+RTER \\
\hline \multirow{3}{*}{ Sentence-level } & Ar+Ch & Ur & 49.9 & 49.1 & 49.5 & 50.1 & 50.1 & 54.5 & $\mathbf{5 5 . 6}$ \\
& Ar+Ur & Ch & 53.9 & 61.1 & 53.1 & 50.3 & 57.3 & 58.0 & $\mathbf{6 2 . 7}$ \\
& Ch+Ur & Ar & 52.5 & 60.1 & 50.4 & 54.5 & 55.2 & 59.9 & $\mathbf{6 1 . 1}$ \\
\hline \multirow{3}{*}{ System-level } & Ar+Ch & Ur & 73.9 & 68.4 & 50.0 & $90.0^{*}$ & $\mathbf{9 2 . 7}^{*}$ & $77.4^{*}$ & $81.0^{*}$ \\
& Ar+Ur & Ch & 38.5 & 44.3 & 40.0 & $\mathbf{5 9 . 0}^{*}$ & $51.8^{*}$ & 47.7 & $57.3^{*}$ \\
& Ch+Ur & Ar & $59.7^{*}$ & $\mathbf{8 6 . 3}^{*}$ & $61.9^{*}$ & 42.1 & 48.1 & $59.7^{*}$ & $61.7^{*}$
\end{tabular}

Table 1: Expt. 1: Spearman's $\rho$ for correlation between human absolute scores and model predictions on NIST OpenMT 2008. Sentence level: All correlations are highly significant. System level: ${ }^{*}: \mathrm{p}<0.05$.

METEORR achieves the best correlation for Chinese and Arabic, but fails for Urdu, apparently the most difficult language. TERR shows the best result for Urdu, but does worse than METEORR for Arabic and even worse than BLEUR for Chinese. The MTR combination metric alleviates this problem to some extent by improving the "worst-case" performance on Urdu to the level of the best individual metric. The entailment-based RTER system outperforms MTR on each language. It particularly improves on MTR's correlation on Urdu. Even though METEORR still does somewhat better than MTR and RTER, we consider this an important confirmation for the usefulness of entailment features in MT evaluation, and for their robustness. ${ }^{5}$

In addition, the combined model MT+RTER is best for all three languages, outperforming METEORR for each language pair. It performs considerably better than either MTR or RTER. This is a second result: the types of evidence provided by MTR and RTER appear to be complementary and can be combined into a superior model.

On the system level (bottom half of Table 1), there is high variance due to the small number of predictions per language, and many predictions are not significantly correlated with human judgments. BLEUR, METEORR, and NISTR significantly predict one language each (all Arabic); TERR, MTR, and RTER predict two languages. MT+RTER is the only model that shows significance for all three languages. This result supports the conclusions we have drawn from the sentence-level analysis.

Further analysis. We decided to conduct a thorough analysis of the Urdu dataset, the most difficult source language for all metrics. We start with a fea-

\footnotetext{
${ }^{5}$ These results are substantially better than the performance our metric showed in the MetricsMATR 2008 challenge. Beyond general enhancement of our model, we attribute the less good MetricsMATR 2008 results to an infelicitous choice of training data for the submission, coupled with the large amount of ASR output in the test data, whose disfluencies represent an additional layer of problems for deep approaches.
}

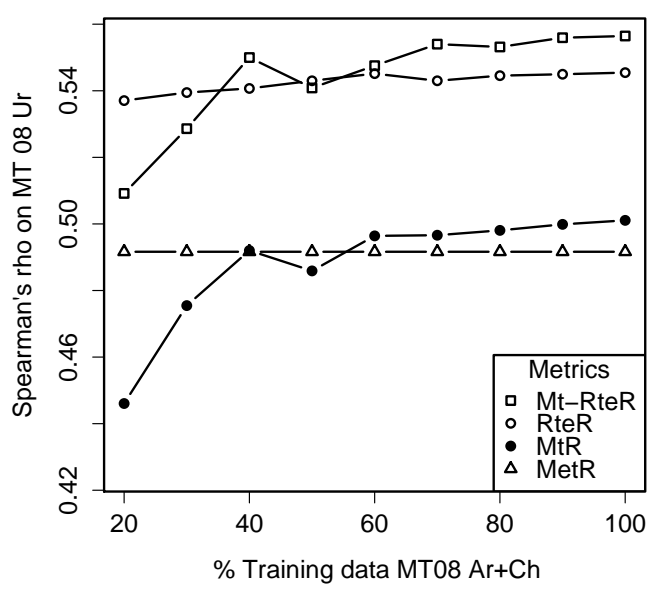

Figure 3: Experiment 1: Learning curve (Urdu).

ture ablation study. Removing any feature group from RTER results in drops in correlation of at least three points. The largest drops occur for the structural $(\delta=-11)$ and insertion/deletion $(\delta=-8)$ features. Thus, all feature groups appear to contribute to the good correlation of RTER. However, there are big differences in the generality of the feature groups: in isolation, the insertion/deletion features achieve almost no correlation, and need to be complemented by more robust features.

Next, we analyze the role of training data. Figure 3 shows Urdu average correlations for models trained on increasing subsets of the training data (10\% increments, 10 random draws per step; Ar and Ch show similar patterns.) METEORR does not improve, which is to be expected given the model definition. RTER has a rather flat learning curve that climbs to within 2 points of the final correlation value for $20 \%$ of the training set (about 400 sentence pairs). Apparently, entailment features do not require a large training set, presumably because most features of RTER are binary. The remaining two models, MTR and MT+RTER, show clearer benefit from more data. With $20 \%$ of the total data, they climb to within 5 points of their final performance, but keep slowly improving further. 
REF: I shall face that fact today.

HYP: Today I will face this reality.

[doc WL-34-174270-7483871, sent 4, system1]
Gold: 6

METEORR: 2.8

RTER: 6.1

- Only function words unaligned (will, this)

- Alignment fact/reality: hypernymy is ok in upward monotone context

REF: What does BBC's Haroon Rasheed say after a visit to Lal Masjid Jamia Hafsa complex? There are no underground tunnels in Lal Masjid or Jamia Hafsa. The presence of the foreigners could not be confirmed as well. What became of the extremists like Abuzar?

HYP: BBC Haroon Rasheed Lal Masjid, Jamia Hafsa after his visit to Auob Medical Complex says Lal Masjid and seminary in under a land mine, not also been confirmed the presence of foreigners could not be, such as Abu by the extremist? [doc WL-12-174261-7457007, sent 2, system2]
Gold: 1

METEORR: 4.5

RTER: 1.2

- Hypothesis root node unaligned

- Missing alignments for subjects

- Important entities in hypothesis cannot be aligned

- Reference, hypothesis differ in polarity

Table 2: Expt. 1: Reference translations and MT output (Urdu). Scores are out of 7 (higher is better).

Finally, we provide a qualitative comparison of RTER's performance against the best baseline metric, METEORR. Since the computation of RTER takes considerably more resources than METEORR, it is interesting to compare the predictions of RTER against METEORR. Table 2 shows two classes of examples with apparent improvements.

The first example (top) shows a good translation that is erroneously assigned a low score by METEORR because (a) it cannot align fact and reality (METEORR aligns only synonyms) and (b) it punishes the change of word order through its "penalty" term. RTER correctly assigns a high score. The features show that this prediction results from two semantic judgments. The first is that the lack of alignments for two function words is unproblematic; the second is that the alignment between fact and reality, which is established on the basis of WordNet similarity, is indeed licensed in the current context. More generally, we find that RTER is able to account for more valid variation in good translations because (a) it judges the validity of alignments dependent on context; (b) it incorporates more semantic similarities; and (c) it weighs mismatches according to the word's status.

The second example (bottom) shows a very bad translation that is scored highly by METEORR, since almost all of the reference words appear either literally or as synonyms in the hypothesis (marked in italics). In combination with METEORR's concentration on recall, this is sufficient to yield a moderately high score. In the case of RTER, a number of mismatch features have fired. They indicate problems with the structural well-formedness of the MT output as well as semantic incompatibility between hypothesis and reference (argument structure and reference mismatches).

\section{Expt. 2: Predicting Pairwise Preferences}

In this experiment, we predict human pairwise preference judgments (cf. Section 4). We reuse the linear regression framework from Section 2 and predict pairwise preferences by predicting two absolute scores (as before) and comparing them. ${ }^{6}$

Data. This experiment uses the 2006-2008 corpora of the Workshop on Statistical Machine Translation (WMT) ${ }^{7}$ It consists of data from EUROPARL (Koehn, 2005) and various news commentaries, with five source languages (French, German, Spanish, Czech, and Hungarian). As training set, we use the portions of WMT 2006 and 2007 that are annotated with absolute scores on a fivepoint scale (around 14,000 sentences produced by 40 systems). The test set is formed by the WMT 2008 relative rank annotation task. As in Experiment 1 , we set $\varepsilon$ so that the incidence of ties in the training and test set is equal (60\%).

Results. Table 4 shows the results. The left result column shows consistency, i.e., the accuracy on human pairwise preference judgments. ${ }^{8}$ The pattern of results matches our observations in Expt. 1: Among individual metrics, METEORR and TERR do better than BLEUR and NISTR. MTR and RTER outperform individual metrics. The best result by a wide margin, $52.5 \%$, is shown by MT+RTER.

\footnotetext{
${ }^{6} \mathrm{We}$ also experimented with a logistic regression model that predicts binary preferences directly. Its performance is comparable; see Padó et al. (2009) for details.

${ }^{7}$ Available from http: //www . statmt .org/.

${ }^{8}$ The random baseline is not $50 \%$, but, according to our experiments, $39.8 \%$. This has two reasons: (1) the judgments include contradictory and tie annotations that cannot be predicted correctly (raw inter-annotator agreement on WMT 2008 was $58 \%$ ); (2) metrics have to submit a total order over the translations for each sentence, which introduces transitivity constraints. For details, see Callison-Burch et al. (2008).
} 


\begin{tabular}{l|l|l|l||l} 
Segment & MTR & RTER & MT+RTER & Gold \\
\hline $\begin{array}{l}\text { REF: Scottish NHS boards need to improve criminal records checks for } \\
\text { employees outside Europe, a watchdog has said. }\end{array}$ & Rank: 3 & Rank: 1 & Rank: 2 & Rank: 1 \\
$\begin{array}{l}\text { HYP: The Scottish health ministry should improve the controls on extra- } \\
\text { community employees to check whether they have criminal precedents, } \\
\text { said the monitoring committee. [1357, lium-systran] }\end{array}$ & & & \\
\hline $\begin{array}{l}\text { REF: Arguments, bullying and fights between the pupils have extended } \\
\text { to the relations between their parents. }\end{array}$ & Rank: 5 & Rank: 2 & Rank: 4 & Rank: 5 \\
$\begin{array}{l}\text { HYP: Disputes, chicane and fights between the pupils transposed in } \\
\text { relations between the parents. [686, rbmt4] }\end{array}$ & & & \\
\hline
\end{tabular}

Table 3: Expt. 2: Reference translations and MT output (French). Ranks are out of five (smaller is better).

\begin{tabular}{l|l|l} 
Feature set & $\begin{array}{l}\text { Consis- } \\
\text { tency }(\%)\end{array}$ & $\begin{array}{l}\text { System-level } \\
\text { correlation }(\rho)\end{array}$ \\
\hline BLEUR & 49.6 & 69.3 \\
METEORR & 51.1 & 72.6 \\
NISTR & 50.2 & 70.4 \\
TERR & 51.2 & 72.5 \\
\hline MTR & 51.5 & 73.1 \\
RTER & 51.8 & $\mathbf{7 8 . 3}$ \\
MT+RTER & $\mathbf{5 2 . 5}$ & 75.8 \\
\hline WMT 08 (worst) & 44 & 37 \\
WMT 08 (best) & 56 & 83 \\
\hline
\end{tabular}

Table 4: Expt. 2: Prediction of pairwise preferences on the WMT 2008 dataset.

The right column shows Spearman's $\rho$ for the correlation between human judgments and tieaware system-level predictions. All metrics predict system scores highly significantly, partly due to the larger number of systems compared (87 systems). Again, we see better results for METEORR and TERR than for BLEUR and NISTR, and the individual metrics do worse than the combination models. Among the latter, the order is: MTR (worst), MT+RTER, and RTER (best at 78.3).

WMT 2009. We submitted the Expt. 2 RTER metric to the WMT 2009 shared MT evaluation task (Padó et al., 2009). The results provide further validation for our results and our general approach. At the system level, RTER made third place (avg. correlation $\rho=0.79$ ), trailing the two top metrics closely ( $\rho=0.80, \rho=0.83$ ) and making the best predictions for Hungarian. It also obtained the second-best consistency score (53\%, best: 54\%).

Metric comparison. The pairwise preference annotation of WMT 2008 gives us the opportunity to compare the MTR and RTER models by computing consistency separately on the "top" (highestranked) and "bottom" (lowest-ranked) hypotheses for each reference. RTER performs about 1.5 percent better on the top than on the bottom hypotheses. The MTR model shows the inverse behavior, performing 2 percent worse on the top hypotheses. This matches well with our intuitions: We see some noise-induced degradation for the entailment features, but not much. In contrast, surface-based features are better at detecting bad translations than at discriminating among good ones.

Table 3 further illustrates the difference between the top models on two example sentences. In the top example, RTER makes a more accurate prediction than MTR. The human rater's favorite translation deviates considerably from the reference in lexical choice, syntactic structure, and word order, for which it is punished by MTR (rank 3/5). In contrast, RTER determines correctly that the propositional content of the reference is almost completely preserved (rank 1). In the bottom example, RTER's prediction is less accurate. This sentence was rated as bad by the judge, presumably due to the inappropriate main verb translation. Together with the subject mismatch, MTR correctly predicts a low score (rank 5/5). RTER's attention to semantic overlap leads to an incorrect high score (rank 2/5).

Feature Weights. Finally, we make two observations about feature weights in the RTER model.

First, the model has learned high weights not only for the overall alignment score (which behaves most similarly to traditional metrics), but also for a number of binary syntacto-semantic match and mismatch features. This confirms that these features systematically confer the benefit we have shown anecdotally in Table 2. Features with a consistently negative effect include dropping adjuncts, unaligned or poorly aligned root nodes, incompatible modality between the main clauses, person and location mismatches (as opposed to general mismatches) and wrongly handled passives. Con- 
versely, higher scores result from factors such as high alignment score, matching embeddings under factive verbs, and matches between appositions.

Second, good MT evaluation feature weights are not good weights for RTE. Some differences, particularly for structural features, are caused by the low grammaticality of MT data. For example, the feature that fires for mismatches between dependents of predicates is unreliable on the WMT data. Other differences do reflect more fundamental differences between the two tasks (cf. Section 3). For example, RTE puts high weights onto quantifier and polarity features, both of which have the potential of influencing entailment decisions, but are (at least currently) unimportant for MT evaluation.

\section{Related Work}

Researchers have exploited various resources to enable the matching between words or $n$-grams that are semantically close but not identical. Banerjee and Lavie (2005) and Chan and $\mathrm{Ng}$ (2008) use WordNet, and Zhou et al. (2006) and Kauchak and Barzilay (2006) exploit large collections of automatically-extracted paraphrases. These approaches reduce the risk that a good translation is rated poorly due to lexical deviation, but do not address the problem that a translation may contain many long matches while lacking coherence and grammaticality (cf. the bottom example in Table 2).

Thus, incorporation of syntactic knowledge has been the focus of another line of research. Amigó et al. (2006) use the degree of overlap between the dependency trees of reference and hypothesis as a predictor of translation quality. Similar ideas have been applied by Owczarzak et al. (2008) to LFG parses, and by Liu and Gildea (2005) to features derived from phrase-structure tress. This approach has also been successful for the related task of summarization evaluation (Hovy et al., 2006).

The most comparable work to ours is Giménez and Márquez (2008). Our results agree on the crucial point that the use of a wide range of linguistic knowledge in MT evaluation is desirable and important. However, Giménez and Márquez advocate the use of a bottom-up development process that builds on a set of "heterogeneous", independent metrics each of which measures overlap with respect to one linguistic level. In contrast, our aim is to provide a "top-down", integrated motivation for the features we integrate through the textual entailment recognition paradigm.

\section{Conclusion and Outlook}

In this paper, we have explored a strategy for the evaluation of MT output that aims at comprehensively assessing the meaning equivalence between reference and hypothesis. To do so, we exploit the common ground between MT evaluation and the Recognition of Textual Entailment (RTE), both of which have to distinguish valid from invalid linguistic variation. Conceputalizing MT evaluation as an entailment problem motivates the use of a rich feature set that covers, unlike almost all earlier metrics, a wide range of linguistic levels, including lexical, syntactic, and compositional phenomena.

We have used an off-the-shelf RTE system to compute these features, and demonstrated that a regression model over these features can outperform an ensemble of traditional MT metrics in two experiments on different datasets. Even though the features build on deep linguistic analysis, they are robust enough to be used in a real-world setting, at least on written text. A limited amount of training data is sufficient, and the weights generalize well.

Our data analysis has confirmed that each of the feature groups contributes to the overall success of the RTE metric, and that its gains come from its better success at abstracting away from valid variation (such as word order or lexical substitution), while still detecting major semantic divergences. We have also clarified the relationship between MT evaluation and textual entailment: The majority of phenomena (but not all) that are relevant for RTE are also informative for MT evaluation.

The focus of this study was on the use of an existing RTE infrastructure for MT evaluation. Future work will have to assess the effectiveness of individual features and investigate ways to customize RTE systems for the MT evaluation task. An interesting aspect that we could not follow up on in this paper is that entailment features are linguistically interpretable (cf. Fig. 2) and may find use in uncovering systematic shortcomings of MT systems.

A limitation of our current metric is that it is language-dependent and relies on NLP tools in the target language that are still unavailable for many languages, such as reliable parsers. To some extent, of course, this problem holds as well for state-of-the-art MT systems. Nevertheless, it must be an important focus of future research to develop robust meaning-based metrics for other languages that can cash in the promise that we have shown for evaluating translation into English. 


\section{References}

Enrique Amigó, Jesús Giménez, Julio Gonzalo, and Lluís Màrquez. 2006. MT Evaluation: Humanlike vs. human acceptable. In Proceedings of $\mathrm{COL}$ ING/ACL 2006, pages 17-24, Sydney, Australia.

Satanjeev Banerjee and Alon Lavie. 2005. METEOR: An automatic metric for MT evaluation with improved correlation with human judgments. In Proceedings of the ACL Workshop on Intrinsic and Extrinsic Evaluation Measures, pages 65-72, Ann Arbor, MI.

Chris Callison-Burch, Miles Osborne, and Philipp Koehn. 2006. Re-evaluating the role of BLEU in machine translation research. In Proceedings of EACL, pages 249-256, Trento, Italy.

Chris Callison-Burch, Cameron Fordyce, Philipp Koehn, Christof Monz, and Josh Schroeder. 2008. Further meta-evaluation of machine translation. In Proceedings of the ACL Workshop on Statistical Machine Translation, pages 70-106, Columbus, OH.

Yee Seng Chan and Hwee Tou Ng. 2008. MAXSIM: A maximum similarity metric for machine translation evaluation. In Proceedings of ACL-08: HLT, pages 55-62, Columbus, Ohio, June.

Ido Dagan, Oren Glickman, and Bernardo Magnini. 2005. The PASCAL recognising textual entailment challenge. In Proceedings of the PASCAL Challenges Workshop on Recognising Textual Entailment, Southampton, UK.

Marie-Catherine de Marneffe, Trond Grenager, Bill MacCartney, Daniel Cer, Daniel Ramage, Chloé Kiddon, and Christopher D. Manning. 2007. Aligning semantic graphs for textual inference and machine reading. In Proceedings of the AAAI Spring Symposium, Stanford, CA.

George Doddington. 2002. Automatic evaluation of machine translation quality using n-gram cooccurrence statistics. In Proceedings of HLT, pages 128132, San Diego, CA.

Jesús Giménez and Lluís Márquez. 2008. Heterogeneous automatic MT evaluation through nonparametric metric combinations. In Proceedings of IJCNLP, pages 319-326, Hyderabad, India.

Sanda Harabagiu and Andrew Hickl. 2006. Methods for using textual entailment in open-domain question answering. In Proceedings of ACL, pages 905912, Sydney, Australia.

Eduard Hovy, Chin-Yew Lin, Liang Zhou, and Junichi Fukumoto. 2006. Automated summarization evaluation with basic elements. In Proceedings of LREC, Genoa, Italy.

David Kauchak and Regina Barzilay. 2006. Paraphrasing for automatic evaluation. In Proceedings of HLTNAACL, pages 455-462.
Phillip Koehn. 2005. Europarl: A parallel corpus for statistical machine translation. In Proceedings of the MT Summit X, Phuket, Thailand.

Chin-Yew Lin and Franz Josef Och. 2004. ORANGE: a method for evaluating automatic evaluation metrics for machine translation. In Proceedings of COLING, pages 501-507, Geneva, Switzerland.

Ding Liu and Daniel Gildea. 2005. Syntactic features for evaluation of machine translation. In Proceedings of the ACL Workshop on Intrinsic and Extrinsic Evaluation Measures, pages 25-32, Ann Arbor, MI.

Bill MacCartney and Christopher D. Manning. 2008. Modeling semantic containment and exclusion in natural language inference. In Proceedings of $\mathrm{COL}$ $I N G$, pages 521-528, Manchester, UK.

Bill MacCartney, Trond Grenager, Marie-Catherine de Marneffe, Daniel Cer, and Christopher D. Manning. 2006. Learning to recognize features of valid textual entailments. In Proceedings of NAACL, pages 41-48, New York City, NY.

Franz Josef Och. 2003. Minimum error rate training in statistical machine translation. In Proceedings of $A C L$, pages 160-167, Sapporo, Japan.

Karolina Owczarzak, Josef van Genabith, and Andy Way. 2008. Evaluating machine translation with LFG dependencies. Machine Translation, 21(2):95119.

Sebastian Padó, Michel Galley, Dan Jurafsky, and Christopher D. Manning. 2009. Textual entailment features for machine translation evaluation. In Proceedings of the EACL Workshop on Statistical Machine Translation, pages 37-41, Athens, Greece.

Kishore Papineni, Salim Roukos, Todd Ward, and WeiJing Zhu. 2002. BLEU: a method for automatic evaluation of machine translation. In Proceedings of ACL, pages 311-318, Philadelphia, PA.

Michael Paul, Andrew Finch, and Eiichiro Sumita. 2007. Reducing human assessment of machine translation quality to binary classifiers. In Proceedings of TMI, pages 154-162, Skövde, Sweden.

Matthew Snover, Bonnie Dorr, Richard Schwartz, Linnea Micciulla, and John Makhoul. 2006. A study of translation edit rate with targeted human annotation. In Proceedings of AMTA, pages 223-231, Cambridge, MA.

Matthew Snover, Nitin Madnani, Bonnie J. Dorr, and Richard Schwartz. 2009. Fluency, adequacy, or HTER? Exploring different human judgments with a tunable MT metric. In Proceedings of the EACL Workshop on Statistical Machine Translation, pages 259-268, Athens, Greece.

Liang Zhou, Chin-Yew Lin, and Eduard Hovy. 2006. Re-evaluating machine translation results with paraphrase support. In Proceedings of EMNLP, pages 77-84, Sydney, Australia. 